International Journal of Pure and Applied Mathematics

Volume 86 No. 4 2013, 689-700

ISSN: 1311-8080 (printed version); ISSN: 1314-3395 (on-line version)

url: http://www.ijpam.eu

doi: http://dx.doi.org/10.12732/ijpam.v86i4.8

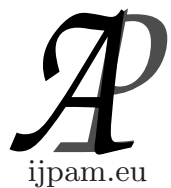

\title{
IZPM FOR UNBALANCED FUZZY \\ TRANSPORTATION PROBLEMS
}

\author{
A. Edward Samuel ${ }^{1}$, M. Venkatachalapathy ${ }^{2}$ \\ ${ }^{1}$ Ramanujan Research Centre \\ P.G. \& Research Department of Mathematics \\ Government Arts College (Autonomous) \\ Kumbakonam, Tamil Nadu, INDIA \\ ${ }^{2}$ Department of Mathematics \\ Oxford Engineering College \\ Tiruchirappalli, Tamil Nadu, INDIA
}

\begin{abstract}
Modifications to Vogel's Approximation Method for obtaining initial solutions to the unbalanced transportation problem are described by many workers. This paper presents a simple and efficient method that is better than the existing methods, easy to understand and also can give an optimal solution. The proposed method, Improved Zero Point Method (IZPM) (see [13]), is used for solving unbalanced fuzzy transportation problems by assuming that a decision maker is uncertain about the precise values of the transportation cost only. In the proposed method transportation costs are represented by triangular fuzzy numbers. The algorithm of the approach is detailed with suitable numerical examples. Further comparative studies of the new technique with other existing algorithms are established by means of sample problems.
\end{abstract}

AMS Subject Classification: 90C08, 90C90, 90C70, 90B06, 90C29 Key Words: unbalanced fuzzy transportation problem, triangular fuzzy number, ranking function, improved zero point method (IZPM)

Received: April 4, 2013

(C) 2013 Academic Publications, Ltd.

${ }^{\S}$ Correspondence author url: www.acadpubl.eu 


\section{Introduction}

In today's highly competitive market, the pressure on organizations is to find better ways to create and deliver value added service to the customers in order to become stronger. Transportation models provide a powerful framework to meet the challenge. They ensure the efficient movement and timely availability of raw materials and finished goods.

The basic transportation problem was originally developed by Hitchcock [16]. The transportation problems can be modeled as a standard linear programming problem, which can then be solved by the simplex method.Several heuristic methods are available to get an initial basic feasible solution [4], [5] and [6]. Although some heuristics can find an initial feasible solution very quickly, oftentimes the solution they find is not very good in terms of minimizing total cost. On the other hand, some heuristics may not find an initial solution as quickly, but the solution they find is often very good in terms of minimizing total cost [2]. There are specialized algorithms for transportation problem that are much more efficient than the simplex algorithm [24]. Wellknown heuristics methods are North West Corner [11], Best Cell Method and Vogel's Approximation Method [23]. Many workers describe modifications to Vogel's Approximation method for obtaining initial solutions to the unbalanced transportation problem. Shimshak [27] propose a modification (SVAM) which ignores any penalty that involves a dummy row/column. For example, if there is a dummy column in the cost-matrix, the penalties are ignored not only for the dummy column, but also for all the rows since the calculation of row-penalties involves the dummy column. Goyal [15] suggests another modification (GVAM) where the cost of transporting goods to or from a dummy point is set equal to the highest transportation cost in the problem, rather than to zero. The method proposed by Ramakrishnan [22] consists of four steps of reduction and one step of VAM. Balakrishnan [21] suggest further modification in SVAM. Kirca and Satir [18] developed a heuristic to obtain efficient initial basic feasible solutions, called Total Opportunity Cost method (TOM). Gass [14] reviewed various methods and discussed on solving the transportation problem. Sharma and Sharma [25] proposed a new procedure to solve the dual of the well-known uncapacitated transportation problem. Sharma and Prasad [26] proposed heuristic gives significantly better solutions than the well-known VAM. This is a best heuristic method than Vogel's to get initial solution to uncapacited transportation problem. Mathirajan and Meenakshi [19] were extended TOM using the VAM procedure. They coupled VAM with total opportunity cost and achieved very efficient initial solutions.Several sorts of methods have been established for 
finding the optimal solution. Among them some methods have been introduced which directly attain at the optimal solution namely zero suffix method [30], ASM-Method [1] etc. But these two methods for finding optimal solution of a transportation problem do not reflect optimal solution proved by Mohammed [20]. In general, the transportation problems are solved with the assumptions that the coefficients or cost parameters are specified in a precise way i.e., in crisp environment.

In real life, there are many diverse situations due to uncertainty in judgments, lack of evidence etc. Sometimes it is not possible to get relevant precise data for the cost parameter. Zimmermann [31] showed that solutions obtained by fuzzy linear programming method is always efficient. Subsequently, Zimmermann's fuzzy linear programming has developed into several fuzzy optimization methods for solving the transportation problems. Chanas and Kuchta [7] proposed the concept of the optimal solution for the transportation problem with fuzzy coefficients expressed as fuzzy numbers, and developed an algorithm for obtaining the optimal solution.

In this study, basic idea is to get an optimal solution for an unbalanced fuzzy transportation problems. This paper presents an algorithm, simple and easy to understand technique for solving both balanced and unbalanced fuzzy transportation problems, which can be an alternative to the modification distribution method/stepping stone method [4], [6]. No path tracing is required in this approach. The proposed method, Improved Zero Point Method (IZPM) (see [13]), is used for solving unbalanced fuzzy transportation problems by assuming that a decision maker is uncertain about the precise values of the transportation cost only. In the proposed method transportation cost are represented by triangular fuzzy numbers. The algorithm of the approach is detailed with suitable numerical examples. Further Comparative studies of the new technique with other existing algorithms are established by means of sample problems.

\section{Proposed Method}

In this section, a proposed method, Improved Zero Point Method [13], for finding an optimal solution using function principle, in which transportation costs are represented as triangular fuzzy number.

The methodology of Improved Zero Point Method (IZPM) (see [13]) is presented as follows:

Step 1. Develop the cost table from the given problem. Construct the fuzzy transportation table for the given fuzzy transportation problem, then 
convert it into a balanced one, if it is not.

Step 2. Find the opportunity cost table. (a) Locate the smallest element in each row of the given cost table and then subtract that from each element of that row, and

(b)In the reduced matrix obtained from 2(a), locate the smallest element in each column and then subtract that from each element of that column. Each row and column have at least one zero value.

Step 3. Optimality criterion. (a) Verify each supply is less than or equal to the total demand, whose reduced costs are zero.

(b) Verify each demand is less than or equal to the total supply, whose reduced costs are zero.

(c) If 3(a) and 3(b) are satisfied, then go to step6 else go to step4.

Step 4. Revise the opportunity cost table. Draw a minimal set of horizontal and vertical lines to cover all the zeros in the opportunity cost table obtained from step2. (Omitting the unsatisfied supply and demand of 3(a)and $3(\mathrm{~b}))$.

Step 5. Develop the new revised opportunity cost table. (a) From among the cells not covered by any line, choose the smallest element. Call this value $\mathrm{k}$.

(b) Subtract $\mathrm{k}$ from every element in the cell not covered by a line.

(c) Add $\mathrm{k}$ to every element in the cell covered by the two lines, i.e., intersection of two lines.

(d) Elements in cells covered by one line remain unchanged.

(e) Go to Step 3.

Step 6. Make assignments in the opportunity cost matrix. The procedure of making assignments is as follows

(a) Identify the largest unit fuzzy transportation cost cell in the cost matrix obtained from either step2 or step5. If there is a tie, use any arbitrary tiebreaking choice. Call this cell as (i,j).

(b) Make the assignment, by selecting ith row and/or jth column with exactly one zero cell.

(c) Adjust the supply and demand and cross out the satisfied row or column.

(d) Repeat this procedure until the entire available supply at various sources and demand at various destinations are satisfied. 


\begin{tabular}{|c|c|c|c|c|}
\hline & D1 & D2 & D3 & Availability(ai) \\
\hline S1 & $(1,2,3)$ & $(4,7,10)$ & $(10,14,18)$ & 5 \\
\hline S2 & $(2,3,4)$ & $(2,3,4)$ & $(0,1,2)$ & 8 \\
\hline S3 & $(1,5,9)$ & $(3,4,5)$ & $(4,7,10)$ & 7 \\
\hline S4 & $(0,1,2)$ & $(5,6,7)$ & $(1,2,3)$ & 15 \\
\hline Demand(bj) & 7 & 9 & 18 & \\
\hline
\end{tabular}

Table 1: The Conventional Solution by VAM, see [23]

\begin{tabular}{|c|c|c|c|c|c|}
\hline & D1 & D2 & D3 & D4 & Availability(ai) \\
\hline S1 & $(1,2,3) \mathbf{5}$ & $(4,7,10)$ & $(10,14,18)$ & $(0,0,0)$ & 5 \\
\hline S2 & $(2,3,4)$ & $(2,3,4)$ & $(0,1,2) \mathbf{8}$ & $(0,0,0)$ & 8 \\
\hline S3 & $(1,5,9)$ & $(3,4,5) \mathbf{6}$ & $(4,7,10)$ & $(0,0,0) \mathbf{1}$ & 7 \\
\hline S4 & $(0,1,2) \mathbf{2}$ & $(5,6,7) \mathbf{3}$ & $(1,2,3) \mathbf{1 0}$ & $(0,0,0)$ & 15 \\
\hline Demand(bj) & 7 & 9 & 18 & 1 & \\
\hline
\end{tabular}

Table 2: The total cost resulting from this initial solution is $\mathbf{8 2}$

\section{Illustrative Example}

To illustrate the proposed method, Improved Zero Point Method(IZPM)[13], is used for solving unbalanced fuzzy transportation problems by assuming that a decision maker is uncertain about the precise values of the transportation costs only.Table1 gives the availability of the product available at four sources and their demand at three destinations, and the approximate cost for transporting one unit quantity of product from each source to each destination is represented by triangular fuzzy number.

Let us consider the following problem, and solve by using the function principle,introduced by chen [28] to treat the fuzzy arithmetical operations and then defuzzify the value by graded mean integration representation method by [29].

\section{Conclusion}

A simple and efficient method that is better than the existing methods, easy to understand and also can give an optimal solution is proposed. The algorithm of 
The Conventional Solution by SVAM [27]

\begin{tabular}{|c|c|c|c|c|c|}
\hline & D1 & D2 & D3 & D4 & Availability(ai) \\
\hline S1 & $(1,2,3) \mathbf{5}$ & $(4,7,10)$ & $(10,14,18)$ & $(0,0,0)$ & 5 \\
\hline S2 & $(2,3,4)$ & $(2,3,4) \mathbf{8}$ & $(0,1,2)$ & $(0,0,0)$ & 8 \\
\hline S3 & $(1,5,9) \mathbf{2}$ & $(3,4,5) \mathbf{1}$ & $(4,7,10) \mathbf{3}$ & $(0,0,0) \mathbf{1}$ & 7 \\
\hline S4 & $(0,1,2)$ & $(5,6,7)$ & $(1,2,3) \mathbf{1 5}$ & $(0,0,0)$ & 15 \\
\hline Demand(bj) & 7 & 9 & 18 & 1 & \\
\hline
\end{tabular}

Table 3

The total cost resulting from this initial solution is $\mathbf{9 9}$.

The Conventional Solution by GVAM [15]

\begin{tabular}{|c|c|c|c|c|c|}
\hline & D1 & D2 & D3 & D4 & Availability(ai) \\
\hline S1 & $(1,2,3) \mathbf{5}$ & $(4,7,10)$ & $(10,14,18)$ & $(0,0,0)$ & 5 \\
\hline S2 & $(2,3,4)$ & $(2,3,4)$ & $(0,1,2) \mathbf{8}$ & $(0,0,0)$ & 8 \\
\hline S3 & $(1,5,9)$ & $(3,4,5) \mathbf{7}$ & $(4,7,10)$ & $(0,0,0)$ & 7 \\
\hline S4 & $(0,1,2) \mathbf{2}$ & $(5,6,7) \mathbf{2}$ & $(1,2,3) \mathbf{1 0}$ & $(0,0,0) \mathbf{1}$ & 15 \\
\hline Demand(bj) & 7 & 9 & 18 & 1 & \\
\hline
\end{tabular}

Table 4

The total cost resulting from this initial solution is $\mathbf{8 0}$.

The Conventional Solution by BVAM [21]

\begin{tabular}{|c|c|c|c|c|c|}
\hline & D1 & D2 & D3 & D4 & Availability(ai) \\
\hline S1 & $(1,2,3) \mathbf{4}$ & $(4,7,10)$ & $(10,14,18)$ & $(0,0,0) \mathbf{1}$ & 5 \\
\hline S2 & $(2,3,4)$ & $(2,3,4)$ & $(0,1,2) \mathbf{8}$ & $(0,0,0)$ & 8 \\
\hline S3 & $(1,5,9)$ & $(3,4,5) \mathbf{7}$ & $(4,7,10)$ & $(0,0,0)$ & 7 \\
\hline S4 & $(0,1,2) \mathbf{3}$ & $(5,6,7) \mathbf{2}$ & $(1,2,3) \mathbf{1 0}$ & $(0,0,0)$ & 15 \\
\hline Demand(bj) & 7 & 9 & 18 & 1 & \\
\hline
\end{tabular}

Table 5

The total cost resulting from this initial solution is $\mathbf{7 9}$. 
The Conventional Solution by RVAM [22]

\begin{tabular}{|c|c|c|c|c|c|}
\hline & D1 & D2 & D3 & D4 & Availability(ai) \\
\hline S1 & $(1,2,3)$ & $(4,7,10) \mathbf{4}$ & $(10,14,18)$ & $(0,0,0) \mathbf{1}$ & 5 \\
\hline S2 & $(2,3,4)$ & $(2,3,4)$ & $(0,1,2) \mathbf{8}$ & $(0,0,0)$ & 8 \\
\hline S3 & $(1,5,9)$ & $(3,4,5) \mathbf{5}$ & $(4,7,10) \mathbf{2}$ & $(0,0,0)$ & 7 \\
\hline S4 & $(0,1,2) \mathbf{7}$ & $(5,6,7)$ & $(1,2,3) \mathbf{8}$ & $(0,0,0)$ & 15 \\
\hline Demand(bj) & 7 & 9 & 18 & 1 & \\
\hline
\end{tabular}

Table 6

The total cost resulting from this initial solution is $\mathbf{9 3}$.

The Conventional Solution by ASM [1]

\begin{tabular}{|c|c|c|c|c|c|}
\hline & D1 & D2 & D3 & D4 & Availability(ai) \\
\hline S1 & $(1,2,3)$ & $(4,7,10)$ & $(10,14,18) \mathbf{4}$ & $(0,0,0) \mathbf{1}$ & 5 \\
\hline S2 & $(2,3,4)$ & $(2,3,4) \mathbf{8}$ & $(0,1,2)$ & $(0,0,0)$ & 8 \\
\hline S3 & $(1,5,9)$ & $(3,4,5) \mathbf{1}$ & $(4,7,10) \mathbf{6}$ & $(0,0,0)$ & 7 \\
\hline S4 & $(0,1,2) \mathbf{7}$ & $(5,6,7)$ & $(1,2,3) \mathbf{8}$ & $(0,0,0)$ & 15 \\
\hline Demand(bj) & 7 & 9 & 18 & 1 & \\
\hline
\end{tabular}

Table 7

The total cost resulting from this initial solution is $\mathbf{1 4 9}$.

The Conventional Solution by ZSM [30]

\begin{tabular}{|c|c|c|c|c|c|}
\hline & D1 & D2 & D3 & D4 & Availability(ai) \\
\hline S1 & $(1,2,3) \mathbf{2}$ & $(4,7,10) \mathbf{2}$ & $(10,14,18)$ & $(0,0,0)$ & 5 \\
\hline S2 & $(2,3,4)$ & $(2,3,4) \mathbf{8}$ & $(0,1,2)$ & $(0,0,0)$ & 8 \\
\hline S3 & $(1,5,9)$ & $(3,4,5) \mathbf{7}$ & $(4,7,10)$ & $(0,0,0)$ & 7 \\
\hline S4 & $(0,1,2) \mathbf{5}$ & $(5,6,7)$ & $(1,2,3) \mathbf{1 0}$ & $(0,0,0)$ & 15 \\
\hline Demand(bj) & 7 & 9 & 18 & 1 & \\
\hline
\end{tabular}

Table 8

The total cost resulting from this initial solution is $\mathbf{7 9}$. 
The Conventional Solution by MODI Method, see [4], [6]

\begin{tabular}{|c|c|c|c|c|c|}
\hline & D1 & D2 & D3 & D4 & Availability(ai) \\
\hline S1 & $(1,2,3) \mathbf{4}$ & $(4,7,10)$ & $(10,14,18)$ & $(0,0,0) \mathbf{1}$ & 5 \\
\hline S2 & $(2,3,4)$ & $(2,3,4) \mathbf{2}$ & $(0,1,2) \mathbf{6}$ & $(0,0,0)$ & 8 \\
\hline S3 & $(1,5,9)$ & $(3,4,5) \mathbf{7}$ & $(4,7,10)$ & $(0,0,0)$ & 7 \\
\hline S4 & $(0,1,2) \mathbf{3}$ & $(5,6,7)$ & $(1,2,3) \mathbf{1 2}$ & $(0,0,0)$ & 15 \\
\hline Demand(bj) & 7 & 9 & 18 & 1 & \\
\hline
\end{tabular}

Table 9

The total cost resulting from this initial solution is $\mathbf{7 5}$.

The Conventional Solution by IZPM [13]

\begin{tabular}{|c|c|c|c|c|c|}
\hline & D1 & D2 & D3 & D4 & Availability(ai) \\
\hline S1 & $(1,2,3)$ & $(4,7,10)$ & $(10,14,18)$ & $(0,0,0)$ & 5 \\
\hline S1 & $(1,2,3) \mathbf{4}$ & $(4,7,10)$ & $(10,14,18)$ & $(0,0,0) \mathbf{1}$ & 5 \\
\hline S2 & $(2,3,4)$ & $(2,3,4) \mathbf{2}$ & $(0,1,2) \mathbf{6}$ & $(0,0,0)$ & 8 \\
\hline S3 & $(1,5,9)$ & $(3,4,5) \mathbf{7}$ & $(4,7,10)$ & $(0,0,0)$ & 7 \\
\hline S4 & $(0,1,2) \mathbf{3}$ & $(5,6,7)$ & $(1,2,3) \mathbf{1 2}$ & $(0,0,0)$ & 15 \\
\hline Demand(bj) & 7 & 9 & 18 & 1 & \\
\hline
\end{tabular}

Table 10

The total cost of this initial solution is $\mathbf{7 5}$, which is in fact optimal.

To illustrate our procedure further, the initial solutions obtained by VAM[23], SVAM[27], GVAM[15], BVAM[21], RVAM[22], ZSM[30], ASM[1], MODI method (see [4], [6]) and our procedure on twelve randomly generated unbalanced fuzzy transportation problems are shown in the following table. The complete details of the twelve problems are not shown here for space considerations, and are available from the author. 
Initial Solutions Obtained by all Procedures

$\begin{array}{cccccccccccc}\text { Prob } & \text { Row } & \text { Col } & \text { VAM } & \text { SVAM } & \text { GVAM } & \text { BVAM } & \text { RVAM } & \text { ASM } & \text { ZSM } & \text { IZPM } & \text { Optimal } \\ 1 & 3 & 3 & 91 & 91 & 91 & 115 & 91 & 91 & 91 & 91 \\ 2 & 3 & 4 & 2424 & 2424 & 2752 & 2464 & 2424 & 2464 & 2424 & 2424 \\ 3 & 3 & 3 & 2600 & 2450 & 2450 & 2450 & 2450 & 2700 & 2450 & 2450 & 2424 \\ 4 & 3 & 4 & 1745 & 1695 & 1665 & 1650 & 1650 & 1745 & 1665 & 1650 \\ 5 & 4 & 5 & 9200 & 9200 & 9200 & 9800 & 9800 & 9300 & 9300 & 9200 & 9200 \\ 6 & 4 & 3 & 515 & 515 & 515 & 515 & 535 & 535 & 515 & 515 & 515 \\ 7 & 4 & 3 & 144 & 183 & 143 & 143 & 143 & 163 & 145 & 143 & 143 \\ 8 & 3 & 4 & 56 & 54 & 50 & 56 & 56 & 54 & 56 & 50 & 50 \\ 9 & 4 & 5 & 1110 & 920 & 940 & 1120 & 1120 & 960 & 1120 & 920 & 920 \\ 10 & 3 & 3 & 487 & 447 & 487 & 696 & 487 & 487 & 487 & 487 & 487 \\ 11 & 4 & 4 & 82 & 99 & 80 & 84 & 93 & 149 & 79 & 75 & 75 \\ 12 & 3 & 5 & 780 & 770 & 770 & 800 & 795 & 780 & 770 & 770 & 770\end{array}$

From the investigations and the results given above, it is clear that proposed method, IZPM[13], is better than VAM[23], SVAM[27], GVAM[15], BVAM[21], RVAM[22], ZSM[30] and ASM[1] for solving unbalanced fuzzy transportation problems and also the solution given by IZPM [13] is an optimal solution, which is equal to MODI method, see [4], [6].

the approach is detailed with suitable numerical examples. Further comparative studies of the new technique with other existing transportation algorithms are established by means of sample problems. The advantage of this method is that it requires very simple arithmetic calculations. This method is very useful for the decision makers to take a decision during uncertainty.So this technique can be applied for solving an unbalanced fuzzy transportation problems occurring in real life situations.

\section{Acknowledgments}

The authors acknowledge the financial support provided by University Grant Commission, Government of India.

\section{References}

[1] Abdul Quddoos, Javaid Shakeel, M.M. Khalid, A new method for finding an optimal solution for transportation problems, International Journal on Computer Science and Engineering, Vol.4 No.07,1271-1274, 2012.

[2] Anderson D.R, Sweeney D.J, Williams T.A, An introduction to management science: quantitative approaches to decision making, Minnesota: West Publishing Company, 1991. 
[3] Amarpreet Kaur, Amit Kumar, A new approach for solving fuzzy transportation problems using generalized trapezoidal fuzzy numbers, Applied soft computing, 1-34, 2011.

[4] Arsham H and Kahn A.B., A simplex-type algorithm for general transportation problems: An alternative to stepping-stone, Journal of Operational Research Society, 40: 581-590, 1989.

[5] Charnes A, Cooper W.W,The stepping-stone method for explaining linear programming calculation in transportation problem, Management Science, 1, 49-69, 1954.

[6] A. Charnes, W.W. Cooper and A. Henderson.,An Introduction to Linear Programming (Wiley, New York, 1953).

[7] Chanas S, Kuchta D, A concept of the optimal solution of the transportation problem with fuzzy cost coefficients, Fuzzy Sets and Systems, 82, 299305, 1996.

[8] Chen S.H., Graded mean integration representation of generalized fuzzy numbers. Journal of The Chinese Fuzzy Systems Association,5(2)(1999c):17.

[9] Chen S.M, Chen J.H, Fuzzy risk analysis based on the ranking generalized fuzzy numbers with different heights and different spreads, Expert Systems with Applications, 36, 6833-6842, 2009.

[10] Chen S.H, Li G.C, Representation, ranking and distance of fuzzy number with exponential membership function using graded mean integration method, Tamsui Oxford Journal of Mathematical Sciences, 16, 125-131, 2000 .

[11] Dantzig G.B,Linear Programming and Extensions, New Jersey: Princeton University Press, 1963.

[12] Edward Samuel A, Venkatachalapathy M, A new dual based approach for the unbalanced Fuzzy Transportation Problem, Applied Mathematical Sciences, 6, 4443-4455, 2012.

[13] Edward Samuel A, Improved Zero Point Method(IZPM) for the Transportation Problems, Applied Mathematical Sciences, 6, 5421 - 5426, 2012.

[14] Gass S.L, On solving the transportation problem, Journal of Operational Research Society, ,41:291-297, 1990. 
[15] Goyal S.K, Improving VAM for unbalanced transportation problems, Journal of Operational Research Society, 35:1113-1114, 1984.

[16] Hitchcock F.L, The distribution of a product from several sources to numerous localities, Journal of Mathematical Physics, 20, 224-230,1941.

[17] Kaufmann A, Gupta M.M,Introduction to Fuzzy Arithmetics: Theory and Applications, New York: Van Nostrand Reinhold, 1991.

[18] Kirca O, Satir A, A heuristic for obtaining an initial solution for the transportation problem, Journal of Operational Research Society, 41(9):865-871, 1990.

[19] Mathirajan M, Meenakshi B, Experimental Analysis of some variants of Vogel's Approximation Method, Asia-pacific Journal of Operational Research, 21(4): 447-462,2004.

[20] Mohammad K.H, Direct Methods for finding optimal solution of a transportation problem are not always reliable, International Refereed Journal of Engineering and Science, Vol.1, Iss.2, 46-52,2012.

[21] Nagaraj Balakrishnan, Modified Vogel's Approximation Method for the Unbalanced Transportation Problem, Applied Mathematics Letter,3: 9-11, 1990.

[22] Ramakrishnan C.S, An Imporvement to Goyal's modified VAM for the unbalanced transportation problem, Journal of Operational Research Society,39: 609-610,1988.

[23] Reinfeld N.V, Vogel W.R, Mathematical Programming, Prentice-Hall, Englewood Cliffs New Jersey, 59-70, 1958.

[24] Winston W.L, Operations Research Applications and Algorithms, California Wadsworth Publishing, 1991.

[25] Sharma R.R.K, Sharma K.D, A new dual based procedure for the transportation problem, European Journal of Operational Research, 122:611$624,2000$.

[26] Sharma R.R.K, Prasad S, Obtaining a good primal solution to the uncapacited transportation problem, European Journal of Operational Research, 144:560-564,2003. 
[27] Shimshak D.G, Kaslik J.Aand Barclay T.K, A modification of Vogel's approximation method through the use of heuristics, Can. J. Opl Res. Inf. Processing, 259-263,1981.

[28] Shan-Huo Chen., Operations on fuzzy numbers with function principle, Tamkang J. Management Sci. 6(1) (1985), pp. 13-25, Taiwan.

[29] Shan-Huo Chen.,Graded mean integration representation of generalized fuzzy numbers, J. Chinese Fuzzy Systems Association 5(2) (1999), 1-7.

[30] Sudhakar V.J, Arunsankar Nand Karpagam T., A new approach for finding optimal solution for transportation problems, European Journal of Scientific Research, Vol.68 No.2, 254-257,2012.

[31] Zimmermann H.J., Fuzzy programming and linear programming with several objective functions, Fuzzy Sets and Systems, 1, 45-55, 1978. 